\title{
Ethical Issues in Suicide Research
}

\author{
Mozaffor $\mathbf{M}^{1}$, Nurunnabi ASM${ }^{2}$, Shahriah $\mathbf{S}^{3}$
}

1. Clinician Researcher, Biomedical Research Foundation (BRF), Dhaka, E Laboratory Consultant and Head, Department of Biochemistry, Ashiyan Medical College Hospital, Dhaka, Bangladesh 2. Graduate student, Dalla Lana School of Public Health, University of Toronto, Ontario, Canada 3. Director, Phoenix Wellness Center, Dhaka, Bangladesh.

E-mail *Corresponding author : miliva17@ yahoo.com

\begin{abstract}
Introduction: Quality research is needed in order to better understand, appropriately respond to, and reduce the incidence of suicide, which must be ethically sound as well. However, in South Asian region, there is a lack of knowledge and clarity around the nature of ethical problems related to suicide research and how to resolve them. This review work aims to describe the possible ethical problems and how to ensure ethical practice in different types of suicide research, especially involving groups of people who are or who have been suicidal.
\end{abstract}

Material And Method: This review was prepared through an extensive searching of published articles in 3 databases - Google, HINARI and PubMed. However, some institutional guidelines were also taken into considerations. Key words used for searching were 'suicide', 'suicidal', 'ethics', 'ethical issues' and 'ethical research'. A total of 18 journal articles and 3 guidelines were finally selected for this review.

Results: Major concerns or ethical issues are accessing the population, potential harm to participants or the researcher, researchers' competency, securing trust and confidence, maintaining confidentiality, providing support to the participants, and responding sensitively to the needs of the family involved.

Conclusion: Ethical research on suicide, especially involving people with suicidal ideation requires both procedures to protect the study participants, and consideration of ethical dilemmas (before, during and after research) as an ongoing negotiated process. The findings of this research provide a collection and compilation of views held by number of researchers, bioethicists, ethics committee members as well as institutions.

Keywords: Suicide, suicidal ideation, ethical issues, suicide research, research ethics

\section{INTRODUCTION}

Suicide constitutes a major public and mental health problem around the world, as near about one million people die due to suicide every year. ${ }^{1}$ Suicide does not just occur in high-income countries; it is a global phenomenon in all regions of the world. In fact, over $79 \%$ of global suicides occurred in low- and middle-income countries in 2016. ${ }^{1}$ Both those who have had history of suicide attempts, or have been bereaved by suicide, have increased the risks of social and mental health problems and suicidal behaviour. $^{2}$ Suicide occurs throughout the lifespan and was the second leading cause of death among 15-29 years age group globally in 2016. ${ }^{1}$ Approximately 90 percent of people who die by suicide have a psychiatric condition such as depression, anxiety or bipolar disorder. ${ }^{3}$ Among all completed suicides, one-half to twothirds are by people who have suffered from mood disorders, sometimes along with alcohol abuse and other dependency problems, ${ }^{3}$ or in people experiencing conflict, disaster, violence, abuse, or loss and a sense of isolation or discrimination, e.g. refugees and migrants; indigenous peoples; lesbian, gay, bisexual, transgender, intersex (LGBTI) persons and prisoners. ${ }^{1,2}$ When both are present, the risk of suicide, of course, increases many folds. ${ }^{2,3}$ Suicide is a very difficult topic to discuss. By far the strongest risk factor for suicide is a previous suicide attempt, hence, silence can have more tragic results, while knowledge and communication can save lives. ${ }^{1,2}$ Education and 
awareness are the keys to suicide prevention, so can be done with timely, evidence-based and often low-cost interventions.1,2 Therefore, quality research is needed in this field in order to better understand, appropriately respond to, and reduce the incidence of suicide, which must be ethically sound as well.,4 By engaging in research, we can work toward understanding suicide and suicidal behavior more fully and thereby uncover and pursue the best ways to reach and treat those who struggle with suicide while providing an evidence base that elevates the issue beyond awareness and moves us toward action. However, in Bangladesh and other South Asian counties, there is a lack of knowledge and clarity around the nature of ethical problems related to suicide research and how to resolve them. This is an inevitable challenge for the researchers, ethics review committee members and institutions involved in such research in approving, conducting and monitoring research on suicide and participants with suicidal ideation. This review aims to describe the possible ethical problems and how to ensure ethical practice in different types of suicide research, especially involving groups of people who are, or who have been suicidal.

\section{MATERIAL AND METHOD}

The review was prepared through an extensive searching of databases between March and July of 2018. The search was confined to Google, HINARI and PubMed published articles. Besides, some institutional guidelines on roles and responsibilities of investigators were taken into consideration. Key words used for searching were 'suicide', 'suicidal', 'ethics', 'ethical issues' and 'ethical research'. We selected the articles and guidelines through the following inclusion criteria:

1. Literature published in English language; 2. Literature published between 2001 and 2018; 3. Articles that discussed ethical issues on suicide (have narratives, or qualitative components and discussion rather than having only quantitative opinions); 4. Ethical issues not conflicting to the cultural norms and values relevant to South Asian region.

Review of literature included both original research and review articles, book chapters and some western professional association's guidelines. Only relevant documents were downloaded after going through the abstracts and undergone further reading. A total of 18 journal articles and 3 guidelines were finally selected for this review.

\section{RESULT}

A total of 21 literature relevant to ethics in suicide research was reviewed, and then findings were aggregated into themes addressing the possible ethical dilemmas associated with suicide research. Finally, discussions and statements were re-synthesized. Major concerns or ethical issues identified are i) accessing the population, ii) potential harm to participants or the researcher, iii) researchers' competency, iv) securing trust and confidence, v) maintaining confidentiality, vi) providing support to the participants, and vii) responding sensitively to the needs of the family involved.

Among those 21 documents, we have categorized based on the ethical issues: i) accessing the population (8), ii) potential harm to participants or the researcher (10), iii) researchers' competency (8), iv) securing trust and confidence (6), v) maintaining confidentiality (8), vi) providing support to the participants (8), and vii) responding sensitively to the needs of the family involved (6). In the following section, several, but not all, crosscutting considerations are discussed, identifying crucial ethical issues that researchers/ethics committee may find useful to address and try to resolve when dealing with a research project involving participants at heightened risk for suicide.

\section{DISCUSSION:}

The safe and ethical design of research studies (accessing the population at heightened risk of suicide, enrolling those participants and asking them the questions about suicidal ideation and/or intentional self-injury in non-suicide focused research) raises unique considerations. ${ }^{4-}$ 11 Methodological questions need to be addressed, as well as practical considerations about the physical environment and clinical setting where study procedures, including risk monitoring and safety planning, should be taking place. ${ }^{5-11}$ However, ethical views or moral arguments may be different among the stakeholders as well. A lack of standardization in how ethics committees assess ethical review 
applications causes different interpretations and assessments of similar applications across committees. For an example, some investigators or researchers may involve individuals of considerable high risk group, i.e. people with suicide ideation, in an intervention study, as because they think that if they exclude those participants, it will seriously hamper the assessment or evaluation of the effectiveness of that intervention for those who most in need. ${ }^{4,10}$ Now the members of one ethics committee may grant the permission feeling the necessity of such research, while the other may require that these participants should be excluded from such study for their safety issues. ${ }^{5}$ In a similar case, another ethics committee may hold the view that asking participants questions about suicidal behaviour or suicide bereavement may increase their risk of non-fatal or fatal suicidal behaviour. ${ }^{5}$ Here, an exercise in balancing safety issues may be compounded by issues stemming from moral views on suicide, related to the (un)acceptability of suicide and the moral obligations of an individual to save a life. ${ }^{7}$

Studies which include suicide risk evaluation with patients in an emergency room or outpatient department will want to determine in advance, and in collaboration with the clinical service providers, how a positive risk screen will be handled so that the respective patient may receive appropriate and timely care regardless of whether he/she participate in the research study.6,11-12 This will eventually prevent any coercion to participate in research. Moreover, the investigators have the responsibility to determine intellectual and mental capacity of the participants to give informed consent, $5-11$ especially in paediatric research (up to 18 years of age in some jurisdictions), whether a minor has the intellectual capacity and maturity to provide assent for participation in an intervention study. ${ }^{12-14}$ Investigators may be able to include suicidal adolescents who are homeless or possibly physically or sexually abused without parent/guardian consent when either involvement of parent or guardian would compromise the safety of those adolescents, or the adolescents have the individual mental and intellectual capacity to give informed consent. ${ }^{12-}$ ${ }^{14}$ However, these types of studies would require special consideration and approval by the ethics review board.

In efficacy studies of any new suicide prevention interventions have the challenge of determining an appropriate comparator arm, as there may be no established effective standard of care within that setting for reducing suicidal ideation and behaviour in the population specified for study. In such situation, ethical questions arise when suicidal individuals of being assigned to the "placebo arm" versus the active intervention, or risks raised by engaging in an untested intervention. ${ }^{6-11}$

The language on confidentiality is golden in all research studies and should not be limited due to concerns about imminent risk of suicide. ${ }^{15-17}$ However, when relevant, informing individuals, and families about the clinical management plans on possible suicide risk is an important aspect of safe and ethical research, ${ }^{4,8,9}$ which must be balanced with confidentiality guidelines, ${ }^{5}$ as to act in the best interest of the study participants. At the end of the study, two vital issues require special attention - a) to assess that individuals are safe and have information that will help them stay safe, and b) assuring that individual are not being "abandoned" without care by providing linkages to care facilities. ${ }^{6-9,15-16}$

A positive experience is perceived during the mental health assessment and thereby, success of suicide research is achieved when participants are convinced about the competence of the researchers and they could be able to gain trust and confidence. $4,7,18,19$ The participants try to look at whether they get a comprehensive care in the research setting, have ample time to respond, are sure of genuineness of the clinician conducting the assessment; and receive an explanation of the rationale and goals of the assessment/research. ${ }^{18,19}$ Besides, the repetitiveness of the same suicide-related questions or worked from same assessment templates questions, and sense of communication gaps among providers may create exhaustion and reluctance among the study participants., $18-20$ They are also concerned that endorsing any suicidal ideation questions might lead to misjudgment about their safety and overreaction by hospitalizing them, perhaps involuntarily psychiatric hospitalization, which 
seems counter-therapeutic to many of the participants. ${ }^{18,19,21}$

Involvement of family members in suicide research is still debatable. The involvement of family and any informal support during suicide risk assessment is determined by several factors. If a person is acutely suicidal, the first responsibility is to protect his or her safety, which may involve breaching confidentiality as required by law and ethical codes of conduct.6,21,22 In contrast, sometimes family worries about suicidality are associated with compromised family physical and mental health and consequently, diminished ability to offer needed support. ${ }^{22}$ Moreover, family caregivers' suicide caring competence is important to prevent their relatives with suicidal tendencies from attempting suicide, ${ }^{23}$ and more critical especially for older adults who are in need. ${ }^{24}$ Therefore, attempts should be taken to involve family caregivers in suicide research and educate them to build up their competences to care for their relatives with suicidal ideations and/or behaviours.., $21-24$

When developing a research design and protocol by working through the above mentioned issues in consultation with respective institutional policies and guidance as well as existing law in this regard in the territory, will tend to promote ethically competent and scientifically robust studies in upcoming days in the field of suicide research and will surely help to strengthen suicide prevention programmes.

\section{CONCLUSION:}

Ethical research on suicide, especially involving people with suicidal ideation requires both procedures to protect the study participants, and consideration of ethical dilemmas (before, during and after research) as an ongoing negotiated process. The findings of this research provide a collection and compilation of views held by number of researchers, bioethicists, ethics committee members as well as institutions. Suicide is a complex issue and therefore, suicide prevention efforts require coordination and collaboration among multiple sectors of the society, along with comprehensive and integrated efforts. Moreover, we expect that, in our South Asian region, hospital authorities, mental health institutes as well as country's medical research council like BMRC, NHRC, ICMR, PMRC etc. should have specific guidelines on this specific field.

ACKNOWLEDGEMENT: None

FUNDING: None

\section{CONFLICT OF INTEREST: None}

\section{REFERENCES:}

1. World Health organization (WHO). Suicide fact sheet. (Accessed on March 07, 2018). Retrieved from: https://www.who.int/news-room/factsheets/detail/suicide.

2. O'Connor RC, Pirkis J. editors. The International Handbook of Suicide Prevention. 2nd ed. WileyBlackwell; Chichester, UK: 2016.

3. Isometsä E. Suicidal behaviour in mood disorders who, when, and why? Can J Psychiatry 2014;59(3):120-30.

4. Fisher CB, Pearson JL, Kim S, Reynolds CF. Ethical issues in including suicidal individuals in clinical research. IRB: Ethics \& Human Research 2002;24(5):9-14.

5. Lakeman R., FitzGerald M. The ethics of suicide research: The views of ethics committee members. Crisis 2009;30(1):13-9.

6. National Institute of Mental Health (NIMH). Conducting research with participants at elevated risk for suicide: Considerations for researchers. (Accessed on March 11, 2018). Retrieved from: https://www.nimh.nih.gov/funding/clinical-

research/conducting-research-with-participants-atelevated-risk-for-suicide-considerations-forresearchers.shtml.

7. Mishara BL, Weisstub DN. Ethical and legal issues in suicide research. Int J Law Psychiatry 2005;28(1):2341.

8. Lakeman $R$, Fitzgerald, $M$. Ethical suicide research: A survey of researchers. Int J Ment Health Nurs 2009;18(1):10-7.

9. Hom MA, Podlogar MC, Stanley IH, Joiner TE. Ethical issues and practical challenges in suicide research. Crisis 2017;38(2):107-14.

10. Mckenzie SK, Li C, Jenkin G, Collings S. Ethical considerations in sensitive suicide research reliant on non-clinical researchers. Research Ethics 2017;13(34):173-83.

11. Pearson JL, Stanley $B$, King $C A$, Fisher $C B$. Intervention research with persons at high risk for suicidality: safety and ethical considerations. J Clin Psychiatry 2001;62(Suppl 25):17-26.

12. King CA, Kramer AC. Intervention research with youths at elevated risk for suicide: meeting the ethical and regulatory challenges of informed consent and assent. Suicide Life Threat Behav 2008;38(5):486-97. 
13. Fisher $C B$. Informed consent and clinical research involving children and adolescents: Implications of the revised APA ethics code and HIPAA. J Clin Child Adolesc Psychol 2004;33(4):832-9.

14. National Institutes of Health (NIH). NIH policy and guidelines on the inclusion of children as participants in research involving human subjects. (Accessed on March 17, 2018). Retrieved from: http://grants.nih.gov/grants/guide/notice-files/not98024.html.

15. Oquendo MA, Stanley B, Ellis SP, Mann JJ. Protection of human subjects in intervention research for suicidal behavior. Am J Psychiatry. 2004;161(9):1558-63.

16. Reynolds SK, Lindenboim N, Comtois KA, Murray A, Linehan MM. Risky assessments: Participant suicidality and distress associated with research assessments in a treatment study of suicidal behavior. Suicide Life-Threat Behav 2006;36(1):19-34.

17. Gibson S, Benson O, Brand SL. Talking about suicide: confidentiality and anonymity in qualitative research. Nurs Ethic 2013;20(1):18-29.

18. Tsao C, Layde JB, Roberts $L W$. A review of ethics in psychiatric research. Curr Opin Psychiatry 2008;21(6):572-7.

19. Ganzini L, Denneson LM, Press N, Bair MJ, Helmer DA, Poat J, Dobscha SK. Trust is the basis for effective suicide risk screening and assessment in veterans. J Gen Intern Med 2013;28(9):1215-21.

20. Kaplan MS, McFarland BH, Huguet N, Valenstein $M$. Suicide risk and precipitating circumstances among young, middle-aged, and older male veterans. Am J Public Health 2012;102(Suppl 1):131-7.

21. American Psychiatric Association (APA). Practice guideline for the assessment and treatment of patients with suicidal behaviors. Am J Psychiatry 2003;160(S11):1-60.

22. Chiang CY, Lu CY, Lin YH, Lin HY, Sun FK. Caring stress, suicidal attitude and suicide care ability among family caregivers of suicidal individuals: A path analysis. $J$ Psychiatr Ment Health Nurs 2015;22(10):792-800.

23. Frey LM, Cerel J. Risk for suicide and the role of family: A narrative review. I Family Issues 2015;36(6):716-36.

24. Corna LM, Cairney J, Streiner DL. Suicide ideation in older adults: Relationship to mental health problems and service use. Gerontologist 2010;50(6):785-97. 